\title{
An Expansion Method for Parabolic Partial Differential Equations
}

\author{
J. W. Green ${ }^{2}$
}

\begin{abstract}
The aim of this paper is to adapt to certain parabolic partial differential equations an expansion method of solution developed by S. Faedo ${ }^{3}$ for hyperbolic equations. In order to make possible a moderately compact presentation, the equations treated are not the most general to which the method is applicable, but are the simplest nontrivial relatives of the heat equation. Similarly, the boundary values and initial conditions are not the most general, but are assumed to be in a canonical form to which others, if sufficiently smooth, can be reduced. The method of solution not only shows the existence of a solution, but describes a definite procedure for approximating it. Some remarks are made on the possibility of estimating the error.
\end{abstract}

\section{Statement of Problem}

In the region $S, 0 \leq x \leq \pi, 0 \leq t<\infty$, we seek a solution of the equation

$$
L(u) \equiv u_{x x}-u_{t}-g u=f
$$

subject to the initial conditions

$$
u(x, 0)=u(0, t)=u(\pi, t)=0 .
$$

The functions $f$ and $g$ are continuous in $S$, together with their first two partial derivatives. As a compatibility condition, we require that $f(0,0)=f(\pi, 0)=0$. It is convenient to assume that $g \geq 0$. This is not at all essential, since a substitution of the form $u=e^{\lambda t} v$ transforms (1) into

$$
v_{x x}-v_{t}-(g+\lambda) v=f e^{-\lambda t} .
$$

The new coefficient $g+\lambda$ can be made positive in as large a part of $S$ as desired, and this is sufficient for our purposes.

This method can be applied to more general equations in which $u_{t}$ has a variable positive coefficient and the $u_{x}$ term is present. However, the equation selected is of sufficient generality to illustrate the method and results and yet not make the computations unpleasantly complicated. In case $g \equiv$ constant, the method reduces to the method of expanding in eigenfunctions.

\section{The Moment Method ${ }^{4}$}

We seek an approximation to $u$ in the form ${ }^{5,6}$

$$
u_{n}(x, t)=\sum_{k} C_{n, k}(t) \sin k x,
$$

\footnotetext{
1 The preparation of this paper was sponsored (in part) by the Office of Naval Research.

2 University of California and National Bureau of Standards.

s S. Faedo, Un nuovo metodo per l'analisi esistenziale e quantitativa dei problemi di propagazioni. Annali della Scuola Normale Superiore di Pisa, [III], 1, Fase. I-IV (1947).

"Or Galerkin's method. See, I. S. Sokolnikoff, Mathematical theory of elasticity (McGraw-Hill Book Co., Inc., New York, N. Y., 1946)。

5 As on p. 37 of the reference given in footnote 3 , after the existence of the solu. tion has been proved, one can show that the system \{sin $k x\}$ can be replaced by other suitable orthonormal systems.

${ }^{6} \Sigma$ means $\Sigma_{1}^{n}$ and $\int$ means $\int_{0}^{\pi}$.
}

subject to

$$
\int\left\{L\left(u_{n}\right)-f\right\} \sin j x d x=0, \quad j=1,2, \ldots, n,
$$

where

$$
C_{n, k}(0)=0 .
$$

Equation (4) amounts to

$$
-j^{2} C_{n, j}(t)-C_{n, j}^{\prime}(t)-\sum_{k} C_{n, k}(t) b_{k, j}(t)-a_{j}(t)=0,
$$

where

$$
\begin{aligned}
b_{k, j}(t) & =\frac{2}{\pi} \int g(x, t) \sin j x \sin k x d x, \\
a_{j}(t) & =\frac{2}{\pi} \int f(x, t) \sin j x d x .
\end{aligned}
$$

The functions $C_{n, j}(t)$ are thus determined by a system of $n$ simultaneous first-order linear differential equations. These equations are already in canonical form, that is, the derivatives are solved for in terms of the other quantities, and so they have a unique solution satisfying (5). Thus the functions $u_{n}(x, t)$ are determined in unique fashion.

We intend to show that the sequence $u_{n}(x, t)$ converges to a solution of (1). The method proceeds by showing that the $u_{n}$ and certain of their derivatives are uniformly equicontinuous. Recall that the functions $f$ of a family are uniformly equicontinuous in a region, provided that $\epsilon$ is given greater than 0 , there is a $\delta>0$ such that $|f(p)-f(q)|<\epsilon$ for any $f$ of the family and any two points $p$ and $q$ of the region whose distance does not exceed $\delta$. Now, Ascoli's theorem asserts that out of a uniformly bounded and equicontinuous sequence of functions a uniformly continuous subsequence can be selected. Therefore, by selecting subsequences of subsequences a sufficient number of times, we shall arrive at a subsequence of the $u_{n}$ which converges uniformly, together with sufficiently many of its derivatives to allow us to make the existence proof. This latter is effected by using a Green's function and showing that the $u_{n}$, for $n \rightarrow \infty$, come 
successively closer to satisfying an integral equation corresponding to (1), and thus that the limit function does satisfy such an equation.

To prove equicontinuity of our sequences, we make use of a criterion of Tonelli ${ }^{7}$ as follows: A class of continuous functions $v(x, t)$ in the region $S_{T}: 0 \leq x \leq \pi$, $0 \leq t \leq T$, is uniformly equicontinuous in this region if (a) for each $\bar{x}$ and $\bar{t}, v(\bar{x}, t)$ and $v(x, \bar{t})$ are absolutely continuous functions of $t$ and $x$, respectively, (b) the partial derivatives $v_{x}$ and $v_{t}$ are summable in $S_{T}$, (c) there exists a constant $c$ such that $\int v_{x}^{2} d x<c$ and $\int v_{t}^{2} d x<c$ for all members of the family. Furthermore any accumulation function of a class satisfying (a), (b), and (c) also satisfies (a) and (b).

Now the functions $u_{n}$ and those partial derivatives in which we are interested are easily seen to be continuous and to satisfy (a) and (b), and the burden of the proof of equicontinuity falls on obtaining inequalities of the form in (c). This is done by making use of devices similar to those frequently employed to show uniqueness, and to recall them, we shall first prove a uniqueness theorem.

\section{Uniqueness}

Suppose that two solutions of the system (1) (2) exist, all derivatives appearing in (1) being continuous in $S$. Their difference $v$ will satisfy (1), (2) with $f$ replaced by zero. Thus $L(v)=0$ and so

$$
\int L(v) v d x=\int\left\{v_{x x}-v_{t}-g v\right\} v d x=0 .
$$

Now

$$
\int v_{t} v d x=\int \frac{1}{2} \frac{d}{d t} v^{2} d x=\frac{1}{2} \frac{d}{d t} \int v^{2} d x
$$

and

$$
\int v_{x x} v d x=\left.v_{x} v\right|_{0} ^{\pi}-\int v_{x}^{2} d x=-\int v_{x}^{2} d x
$$

If we put $K(t)=\int v^{2} d x$, (7) becomes

$$
\frac{1}{2} K^{\prime}(t)=-\mathcal{S}\left(v_{x}^{2}+g v^{2}\right) d x \leq 0,
$$

since $g \geq 0$. Since by $(2), K(0)=0$, we see that $K(t) \leq 0$. But $K$ is by its nature nonnegative and $K \equiv 0$, which implies that $v \equiv 0$ and that the two solutions are identical.

Theorem 0. There is at most one function, continuous together with all derivatives appearing in (1) which satisfies the system (1), (2).

\section{Certain Bounds}

This section is devoted to obtaining the inequalities required to carry out the program outlined in section 3 .

4.1. Lemma. Let $K(x)$ be a nonnegative function satisfying for $x \geq 0$ the inequality $K^{\prime}(x) \leq \alpha \sqrt{K(x)}$, where $\alpha \geq 0$. Then for $x>0, \sqrt{K(x)} \leq \sqrt{K(0)}+(\alpha / 2) x$.

\footnotetext{
7 Tonelli, L'estremo assoluto degli integrali doppi, Annali della Scuola Normale
} di Pisa (1933).
Suppose that for some positive $x_{0}, \sqrt{K\left(x_{0}\right)}>$ $\sqrt{K(0)}+(\alpha / 2) x_{0}$. Then there exists $x_{1}$ such that $\sqrt{K\left(x_{1}\right)}=\sqrt{K(0)}+(\alpha / 2) x_{1}$, but $\sqrt{K(x)}>\sqrt{K(0)}+(\alpha / 2) x$ for $x_{1}<x \leq x_{0}$. In particular, $K(x)>0$ in $x_{1}<x<x_{0}$, and so in that interval

$$
\frac{K^{\prime}(x)}{\sqrt{K(x)}} \leq \alpha .
$$

Integrating from $x_{1}$ to $x_{0}$, we have

$$
\sqrt{K\left(x_{0}\right)}-\sqrt{K\left(x_{1}\right)} \leq(\alpha / 2)\left(x_{0}-x_{1}\right),
$$

or

$$
\sqrt{K\left(x_{0}\right)} \leq \sqrt{K\left(x_{1}\right)}+(\alpha / 2)\left(x_{0}-x_{1}\right)=\sqrt{K(0)}+(\alpha / 2) x_{0},
$$

a contradiction. The lemma is proved.

Corollary. Let $K(x)$ be a nonnegative function satisfying for $x \geq 0$ the inequality

$$
K^{\prime}(x) \leq \alpha(x) \sqrt{K(x)}
$$

where $\alpha(x)$ is nonnegative and nondecreasing. Then for $x \geq 0$,

$$
\sqrt{K(x)} \leq \sqrt{K(0)}+\frac{1}{2} x \alpha(x) .
$$

Consider any $x_{0}>0$. For $x \leq x_{0}, K^{\prime}(x) \leq \alpha\left(x_{0}\right) \sqrt{K(x)}$. By the preceeding lemma, $\sqrt{K(x)} \leq \sqrt{K(0)}+\frac{1}{2} \alpha\left(x_{0}\right) x$. Setting $x=x_{0}$ and noting that $x_{0}$ is arbitrary, we get the stated result.

4.2. The bound ${ }^{8}$ for $\int u_{n}^{2} d x$. Let each of the equations (4) be multiplied by $C_{n, j}(t)$ and the results summed. There comes out the equation

$$
\int\left\{u_{x x}-u_{t}-g u-f\right\} u d x=0 .
$$

Since

$$
\int u_{x x} u d x=-\int u_{x}^{2} d x \text { and } \int u_{t} u d x=\frac{1}{2} \frac{d}{d t} \int u^{2} d x,
$$

(10) may be written

$$
\frac{1}{2} \frac{d}{d t} \int u^{2} d x=-\int\left(u_{x}^{2}+g u^{2}\right) d x-\int f u d x \leq \mathcal{S}|f u| d x .
$$

Set $\int u^{2}(x, t) d x=K(x)$. From (11) and Schwarz's inequality,

$$
\frac{1}{2} K^{\prime}(x) \leq \mathcal{S}|f u| d x \leq \sqrt{\int f^{2} d x} \cdot \sqrt{\int u^{2} d x}
$$

Set

$$
\max _{\tau \leq t} \sqrt{\int f^{2}(x, \tau) d x}=F(t)
$$

Then (12) gives $K^{\prime}(t) \leq 2 F(t) \sqrt{K(t)}$. From the corollary of 4.1 we deduce that $\sqrt{K(t)} \leq \sqrt{K(0)}+t F(t)$. Now $K(0)=\int u^{2}(x, 0) d x=0$, and so $\sqrt{K(t)} \leq t F(t)$.

${ }^{8}$ In section 4 we shall frequently omit the subscript $n$ from $u_{\text {s. }}$ Whenever $u$ appears in section 4 , it will mean $u_{n}$. 
Theorem 1.

$$
\int u_{n}^{2}(x, t) d x \leq t^{2} F^{2}(t)
$$

where $F$ is given by (13).

4.3. The bound for $\int u_{n t}^{2} d x$. Let the equations (4) be differentiated with respect to $t$, multiplied by $C_{n, i}^{\prime}(t)$, and added. There results from this

$$
\int\left\{u_{x x t}-u_{t t}-g u_{t}-g_{t} u-f_{t}\right\} u_{t} d x=0 .
$$

Again,

$$
\int u_{x x t} u_{t} d x=-\int u_{x t}^{2} d x \text { and } \int u_{t t} u_{t} d t=\frac{1}{2} \frac{d}{d t} \int u_{t}^{2} d x
$$

so putting $K(t)=\int u_{t}^{2} d x$, we get

$$
\begin{aligned}
\frac{1}{2} K^{\prime}(t) & =-\mathcal{S}\left(u_{x t}^{2}+g u_{t}^{2}\right) d x-\mathcal{S}\left(g_{t} u+f_{t}\right) u_{t} d x \\
& \leq \mathcal{S}\left(\left|g_{t} u\right|+\left|f_{t}\right|\right)\left|u_{t}\right| d x \\
& \leq \sqrt{\mathcal{S}\left(\left|g_{t} u\right|+\left|f_{t}\right|\right)^{2}} d x \cdot \sqrt{K(t)} .
\end{aligned}
$$

We define

$$
\max _{\tau \leq t}\left|g_{t}(x, t)\right|=G_{1}(t)
$$

and

$$
\max _{\tau \leq t} \sqrt{\int f_{t}^{2}(x, \tau) d x}=F_{1}(t) .
$$

Then

$$
\begin{aligned}
\mathcal{S}\left(\left|g_{t} u\right|\right. & \left.+\left|f_{t}\right|\right)^{2} d x \leq \mathcal{S}\left(G_{1}^{2}(t) u^{2}+2 G_{1}(t)\left|u f_{t}\right|+f_{t}^{2}\right) d x \\
& \leq G_{1}^{2}(t) \mathcal{S} u^{2} d x+2 G_{1}(t) \sqrt{\mathcal{S} u^{2} d x \mathcal{S} f_{t}^{2} d x}+\int f_{t}^{2} d x \\
& \leq G_{1}^{2}(t) t^{2} F^{2}(t)+2 G_{1}(t) t F(t) F_{1}(t)+F_{1}^{2}(t)=H^{2}(t),
\end{aligned}
$$

this last equation being a definition of $H(t)$.

From (16) we have $K^{\prime}(t) \leq 2 H(t) \sqrt{K(t)}$, and by 4.1,

Now

$$
\sqrt{K(t)} \leq \sqrt{K(0)}+t H(t)
$$

$$
\begin{aligned}
K(0) & =\int u_{t}^{2}(x, 0)=\mathcal{S}\left(\sum C_{n, k}^{\prime}(0) \sin k x\right)^{2} d x \\
& \left.=\frac{\pi}{2} \sum C_{n, k}^{\prime}(0)\right)^{2}=\frac{2}{\pi} \sum\left(\int f(x, 0) \sin k x d x\right)^{2} \\
& \leq \mathcal{S} f^{2}(x, 0) d x
\end{aligned}
$$

by Bessel's inequality. Thus $K(0) \leq F^{2}(0)$, and from (19) we conclude that $\sqrt{K(t)} \leq F(0)+t H(t)$.

\section{Theorem 2.}

$$
\int u_{n t}^{2}(x, t) d x \leq(F(0)+t H(t))^{2}=A^{2}(t),
$$

where $F$ is given by (13),

$$
H^{2}(t)=G_{1}^{2}(t) t^{2} F^{2}(t)+2 G_{1}(t) t F(t) F_{1}(t)+F_{1}^{2}(t),
$$

and $G_{1}$ and $F_{1}$ are given by (17) and (18).
4.4. The bound of $\int u_{n x}^{2} d x$. Equation 10 may be written

$$
\mathcal{S}\left\{-u_{x}^{2}-u u_{t}-g u^{2}-f u\right\} d x=0,
$$

or

$$
\begin{aligned}
\int u_{x}^{2} d x & =-\int\left\{g u^{2}+u u_{t}+f u\right\} d x \leq \mathcal{S}\left(\left|u u_{t}\right|+|f u|\right) d x \\
& \leq \sqrt{\mathcal{S} u^{2} d x \cdot \mathcal{S} u_{t}^{2} d x}+\sqrt{\mathcal{S} u^{2} d x \cdot \int f^{2} d x} \\
& \leq \sqrt{t^{2} F^{2}(t) A^{2}(t)}+\sqrt{t^{2} F^{2}(t) F^{2}(t)} \\
& =t F(t)(A(t)+F(t)) .
\end{aligned}
$$

Theorem 3.

$$
\int u_{n x}^{2}(x, t) d x \leq t F(t)(A(t)+F(t))=B(t),
$$

where $A$ is given by (20).

4.5 The bound of $\int u_{n t t}^{2}(x, t) d x$. We differentiate (4) twice with respect to $t$, multiply by $C_{n i}^{\prime \prime}(t)$ and sum, to get

$$
\mathcal{S}\left\{u_{x x t t}-u_{t t t}-g u_{t t}-2 g_{t} u_{t}-g_{t t} u-f_{t t}\right\} u_{t t}=0 .
$$

As before, we write

$$
\begin{aligned}
& \int u_{x x t} u_{t t} d x=-\int u_{x t t}^{2} d x, \\
& \int u_{t t i} u_{t t} d x=\frac{1}{2} \frac{d}{d t} \int u_{t t}^{2} d x,
\end{aligned}
$$

and (23) becomes, on setting $K(t)=\int u_{t t}^{2} d x$,

$$
\begin{aligned}
\frac{1}{2} K^{\prime}(t) & =-\mathcal{S}\left\{u_{x t}^{2}+g u_{t t}^{2}\right\} d x-\mathcal{S}\left\{2 g_{t} u_{t}+g_{t t} u+f_{t t}\right\} u_{t t} d x \\
& \leq \mathcal{S}\left\{\left|2 g_{t} u_{t}\right|+\left|g_{t t} u\right|+\left|f_{t t}\right|\right\}\left|u_{t t}\right| d x .
\end{aligned}
$$

It is clear that by the use of Schwarz's inequality we can obtain an inequality of the form

$$
K^{\prime}(t) \leq J(t) \sqrt{K(t)}
$$

where $J(t)$ is an increasing and continuous function expressible in terms of $G_{1}, F, F_{1}$, and the similar functions $G_{2}$ and $F_{2}$ corresponding to $g_{t t} f_{t t}$.

We estimate $K(0)$ as follows. Differentiate (4) with respect to $t$ and put $t=0$, to obtain

$$
\begin{aligned}
\int u_{t t}(x, 0) \sin j x d x=\int\left\{u_{x x t}(x, 0)-g u_{t}(x, 0)\right. \\
\\
\left.\quad-f_{t}(x, 0)\right\} \sin j x d x .
\end{aligned}
$$

for $j=1,2, \ldots, n$. Now $u_{t t}(x, 0)$ is a linear combination of $\sin j x, j=1,2, \ldots n$, and by (25) its first $n$ Fourier coefficients are the same as those of $u_{x x t}(x, 0)-g u_{t}(x, 0)-f_{t}(x, 0)$. Thus by Bessel's inequality,

$$
\int u_{t t}^{2}(x, 0) d x \leq \mathcal{S}\left\{u_{x x t}(x, 0)-g u_{t}(x, 0)-f_{t}(x, 0)\right\}^{2} d x .
$$

If in (4) one puts $t=0$, there results

$$
-\int u_{t}(x, 0) \sin j x d x=\int f(x, 0) \sin j x d x .
$$


Two integrations by parts gives

$-\int u_{x x}(x, 0) \sin j x d x=\int f_{x x}(x, 0) \sin j x d x$,

and since $u_{x x t}(x, 0)$ is a linear combination of $\sin j x$, $j=1,2, \ldots, n$, we see as above,

$$
\int u_{x x t}^{2}(x, 0) d x \leq \int f_{x x}^{2}(x, 0) d x .
$$

By using Schwarz's inequality, (27), and the bounds previously obtained, the right hand number of (25) can be dominated by a continuous function depending only on $f$ and $g$ and their derivatives. That is, $K(0)$ is bounded uniformly in $n$, and from (24) it follows that the same is true of $K(t)$.

Theorem 4. There exists a continuous function $C(t)$, determined by $f, g$ and their derivatives, such that

$$
\int u_{n t t}^{2}(x, t) d x \leq C(t) .
$$

4.6. The bound for $\int u_{n x t}^{2} d x$. From eq (15) we have on replacing $\int u_{x x t} u_{t} d x$ by $-\int u_{x t}^{2} d x$,

$$
\int u_{x t}^{2} d x=\int\left\{u_{t t}+g u_{t}+g_{t} u+f_{t}\right\} u_{t t} d x .
$$

Because of the bounds previously obtained, it is clear that we have the theorem:

Theorem 5. There exists a continuous function $D(t)$, expressible in terms of $f$ and $g$ and their derivatives such that

$$
\int u_{n x t}^{2}(x, t) d x \leq D(t) .
$$

4.7. The bound for $\int u_{n x x}^{2} d x$. If equations (4) be multiplied by $j^{2} \sin j x$ and summed, we have

$$
\begin{aligned}
\int u_{x x}^{2} d x & =\mathcal{S}\left\{u_{t}+g u+f\right\} u_{x x} d x \\
& \leq \sqrt{\mathcal{S}\left\{u_{t}+g u+f\right\}^{2} d x} \sqrt{\mathcal{S} u_{x x}^{2} d x} .
\end{aligned}
$$

Now from the previous bounds we can find a continuous function $E(t)$ which depends only on $f, g$ and their derivatives, such that

$$
\mathcal{S}\left\{u_{t}+g u+f\right\}^{2} d x \leq E(t) .
$$

Thus we have the theorem:

Theorem 6. There exists a continuous function $E(t)$ independent of $n$ such that

$$
\int u_{n x x}^{2}(x, t) d x \leq E(t) .
$$

4.8. An integral inequality. Consider the Green's function $G(x, \xi)$ relative to the equation $y^{\prime \prime}+\lambda y=0$ $=y(0)=y(\pi)$. Specifically

$$
G(x, \xi)= \begin{cases}\frac{x(\pi-\xi)}{\pi} & x \leq \xi, \\ \frac{\xi(\pi-x)}{\pi} & x \geq \xi,\end{cases}
$$

and its uniformly convergent Fourier expansion is

$$
G(x, \xi)=-\frac{2}{\pi} \sum_{1}^{\infty} \frac{\sin j x \sin j \xi}{j^{2}} .
$$

For brevity, set

then

$$
u_{\imath}+g u+f=\Phi(u) \text {. }
$$

$$
u_{x x}-\Phi(u)=L(u)-f .
$$

Multiply (34) by $G(x, \xi)$ and integrate with respect to $x$ to obtain

$$
-u(\xi, t)-\int \Phi(u) G(x, \xi) d x=\int\{L(u)-f\} G(x, \xi) d x .
$$

Now

$$
\begin{aligned}
\boldsymbol{\int}\{L(u)-f\} \boldsymbol{G}(x, \xi) d x & \\
& =-\frac{2}{\pi} \sum_{1}^{\infty} \frac{\sin j \xi}{j^{2}} \mathcal{S}\{L(u)-f\} \sin j x d x, \\
& =-\frac{2}{\pi} \sum_{n+1}^{\infty} \frac{\sin j \xi}{j^{2}}\{L(u)-f\} \sin j x d x,
\end{aligned}
$$

because of (4). Now $L(u)-f=u_{x x}-\Phi(u)$, and

$u_{x x}=-\sum_{1}^{n} C_{n, j}(t) j^{2} \sin j x$; hence

$$
\int u_{x x} \sin j x d x=0
$$

for $j>n$, giving

$\mathcal{S}\{L(u)-f\} G(x, \xi) d x=-\frac{2}{\pi} \sum_{n+1}^{\infty} \frac{\sin j \xi}{j^{2}} \mathcal{S} \Phi(u) \sin j x d x$.

By Schwarz's inequality,

$|\boldsymbol{S}\{L(u)-f\} G(x, \xi) d x|$

$$
\leq \frac{2}{\pi} \sqrt{\sum_{n+1}^{\infty} \frac{1}{j^{4}}} \cdot \sqrt{\sum_{n+1}^{\infty}\left(\int \Phi(u) \sin j x d x\right)^{2}} .
$$

Now

$$
\sum_{n+1}^{\infty} \frac{1}{j^{4}} \leq \frac{1}{3 n^{3}}
$$

and

$\sum_{n+1}^{\infty}\left(\int \Phi(u) \sin j x d x\right)^{2}$

$$
\leq \sum_{1}^{\infty}\left(\int \Phi(u) \sin j x d x\right)^{2} \leq \frac{\pi}{2} \int \Phi^{2}(u) d x .
$$

By (30), $\int \Phi^{2}(u) d x \leq E(t)$, and so from (36) we can conclude

$$
|\mathcal{S}\{L(u)-f\} G(x, \xi) d x| \leq \frac{2}{\pi} \sqrt{\frac{1}{3 n^{3}}} \cdot \sqrt{\frac{\pi}{2} E(t)} .
$$

Referring back to (35) we have a theorem:

Theorem 7.

$$
\left|-u_{n}(\xi, t)-\int \Phi\left(u_{n}\right) G(x, \xi) d x\right| \leq \sqrt{\frac{2 E(t)}{3 \pi n^{3}}} .
$$




\section{Existence Theorem}

Recall now the discussion of equicontinuity and the criterion of Tonelli given at the end of section 2 . Because of the inequalities contained in theorems 1 through 6 , we see that the functions $u_{n}, u_{n x}$, and $u_{n t}$ are uniformly equicontinuous. Thus by Ascoli's theorem, we can pick out, by a suitable diagonal process, a subsequence $\left\{v_{m}\right\}$ of $\left\{u_{n}\right\}$ such that

$$
\begin{aligned}
v_{m}(x, t) & \rightarrow u(x, t), \\
v_{m x}(x, t) & \rightarrow v(x, t), \\
v_{m t}(x, t) & \rightarrow w(x, t),
\end{aligned}
$$

the approach being uniform in each case. Because of this uniformity, one sees that $v=\partial u / \partial x$ and $w=\partial u / \partial t$. The derivatives $u_{x x}, u_{x t}, u_{t \imath}$ exist almost everywhere and are summable. We consider the expression

$$
-v_{m}(\xi, t)-\int \Phi\left(v_{m}\right) G(x, \xi) d x .
$$

Since $v_{m}=u_{n_{m}}$, theorem (7) tells us that the expression (38) tends to zero as $m \rightarrow \infty$. Also $\Phi\left(v_{m}\right) \rightarrow \phi(u)$ uniformly, and $G$ is bounded, so we have

$$
\begin{aligned}
\lim _{m \rightarrow \infty}\left\{-v_{m}(\xi, t)-\int \Phi\left(v_{m}\right)\right. & G(x, \xi) d \xi\} \\
& =-u(\xi, t)-\int \Phi(u) G(x, \xi) d x=0 .
\end{aligned}
$$

The integral $\int \Phi(u) G(x, \xi) d x$ has a second derivative with respect to $x$, namely, $-\Phi(u)=-u_{t}-g u-f$. Hence $u_{x x}$ exists, is continuous, and satisfies

$$
-u_{x x}(\xi, t)+\Phi(u)=0,
$$

which is identical with (1). Since $u$ obviously satisfies (2), it is a solution of the system (1), (2) in the region $S_{T}$. But $T$ is arbitrary, and a solution $u$ of (1), (2) exists in all $S$.

We have shown that a subsequence of $u_{n}$ converges to the solution $u$, together with first order derivatives. Actually the entire sequence $u_{n}$ so converges. If this were not the case, there would be a subsequence $u_{n_{j}}$ such that, for instance, $\lim _{n_{j} \rightarrow \infty} u_{n_{j}} \neq u$ at some point $x, t$. From the sequence $u_{n_{j}}$ we could pick another subsequence in the manner of this section, such that this subsequence has for limit a solution $v$ of the problem. By the uniqueness theorem, $u=v$, and so $\lim u_{n_{j}}(x, t)=u(x, t)$, a contradiction. Thus we have

Theorem 8. The sequence $u_{n}(x, t)$ has for uniform limit in any $S_{T}$ the unique function $u(x, t)$ satisfying (1) and (2). Furthermore, $u_{n x} \rightarrow u_{x}$ and $u_{n t} \rightarrow u_{t}$ uniformly in $S_{T}$.

\section{Convergence in Mean of $u_{n \times x}$}

The solution $u(x, t)$ of the system (1), (2) has a Fourier series

$$
u(x, t)=\sum_{1}^{\infty} \lambda_{j}(t) \sin j x
$$

which may be differentiated twice with respect to $x$, and the resulting series converges in the mean to $u_{x x}$. Set

$$
v_{n}=\sum_{1}^{n} \lambda_{j}(t) \sin j x
$$

If (4) are multiplied by $-j^{2}\left(C_{n, j}(t)-\lambda_{j}(t)\right)$ and summed, we get

$$
\mathcal{S}\left\{L\left(u_{n}\right)-f\right\} \frac{\partial^{2}}{\partial x^{2}}\left(u_{n}-v_{n}\right) d x=0 .
$$

Since $L(u)=f$, we may write $(41)$ as

$$
\mathcal{S}\left\{L\left(u_{n}-v_{n}\right)-L\left(u-v_{n}\right)\right\} \frac{\partial^{2}}{\partial x^{2}}\left(u_{n}-v_{n}\right) d x=0 .
$$

Recalling that

$$
L(u)=u_{x x}-u_{t}-g u-f+f=u_{x x}-\Phi(u)+f,
$$

we write (42) in the form

$$
\begin{aligned}
\mathcal{S} & \left\{\frac{\partial^{2}}{\partial x^{2}}\left(u_{n}-v_{n}\right)\right\}^{2} d x \\
& =\mathcal{S}\left\{-\Phi\left(u_{n}-v_{n}\right)+f-L\left(u-v_{n}\right)\right\} \frac{\partial^{2}}{\partial x^{2}}\left(u_{n}-v_{n}\right) d x,
\end{aligned}
$$

and Schwarz's inequality gives

$$
\begin{aligned}
& \sqrt{\int\left\{\frac{\partial^{2}}{\partial x^{2}}\left(u_{n}-v_{n}\right)\right\}^{2}} d x \\
& \quad \leq \sqrt{\mathcal{S}\left\{-\Phi\left(u_{n}-v_{n}\right)+f-L\left(u-v_{n}\right)\right\}^{2}} d x .
\end{aligned}
$$

Now, $-\Phi\left(u_{n}-v_{n}\right)+f=-\left(u_{n}-v_{n}\right)_{t}-g\left(u_{n}-v_{n}\right) \rightarrow 0$ uniformly, and $L\left(v-v_{n}\right)$ tends to zero in the mean; thus the right hand member of (43) tends to zero and so

$$
\mathcal{S}\left\{\frac{\partial^{2}}{\partial x^{2}}\left(u_{n}-v_{n}\right)\right\}^{2} d x \rightarrow 0 .
$$

From this and an appropriate triangular inequality it follows that

$$
\mathcal{S}\left\{\frac{\partial^{2}}{\partial x^{2}}\left(u_{n}-u\right)\right\}^{2} d x \rightarrow 0 .
$$

Theorem 9. The second derivatives $u_{n x x}$ of the approximating functions tend in the mean to $u_{x x}$.

It is too much to expect that $u_{n x x} \rightarrow u_{x x}$ uniformly, or even pointwise. For example, $u_{n x x}(0, t) \equiv 0$, while $u_{x x}(0, t)=f(0, t)$, and this latter does not need to vanish identically.

\section{Estimating the Error}

From theorem 9 it is easy to prove that $L\left(u_{n}\right) \rightarrow L(u)$ in the mean. Set $L\left(u_{n}\right)=f_{n}$. Then

$$
L\left(u_{n}-u\right)=L\left(u_{n}\right)-L(u)=f_{n}-f \stackrel{\text { l.m. }}{\rightarrow} 0 .
$$


Using the method of 4.2 , we prove that

where

$$
\mathcal{S}\left(u_{n}-u\right)^{2} d x \leq t^{2} F_{n}^{* 2}(t),
$$

$$
F_{n}^{*}(t)=\max _{\tau \leq t} \sqrt{\mathcal{S}\left\{f(x, \tau)-f_{n}(x, \tau)\right\}^{2}} d x .
$$

Theorem 10. The mean square error satisfies the inequality

$$
\mathcal{S}\left(u_{n}-u\right)^{2} d x \leq t^{2} F_{n}^{* 2}(t),
$$

where $F_{n}^{*}(t)$ is given by (44), and $F_{n}^{*}(t) \rightarrow 0$ as $n \rightarrow \infty$.

Theorem 10 unfortunately gives information only about the mean square of the error instead of about the error at a point, which would be desirable. This latter will be mentioned below. Apart from this, however, theorem 10 gives a rather curious sort of information about the m.s.e. It does not give an a priori estimate of this error; that is, we cannot say that after $n$ steps the error will have a certain bound, because we do not know how rapidly $F_{n}$ tends to zero. On the other hand, we do seem to know more than the mere fact that the m.s.e. tends to zero. We know that if we take $n$ sufficiently large, the m.s.e. will be small and we shall know it to be small. What we do not know is when we shall know it!

In section 5 it was mentioned that $u_{x t}$ is a summable function. Thus from $L(u)=f$, it follows that $u_{x x t}$ is summable. From the equation $L\left(u_{n}-u\right)$ $=f_{n}-f$, we obtain, using the methods of sections 4.3 and 4.4 , the inequality analogous to $(22)$,

$$
\mathcal{S}\left\{\left(u_{n}-u\right)_{x}\right\}^{2} d x \leq t F_{n}^{*}(t)\left(A_{n}^{*}(t)+F_{n}^{*}(t)\right)=G_{n}^{*(t)},
$$

where $F_{n}^{*}$ and $A_{n}^{*}$ are given by (13) and (20), except that $f_{n}-f$ is used insted of $f$ in their definitions. Now we are unable to say much about the factor $A_{n}^{*}$ since it contains terms of the form $\int\left\{f_{n t}-f_{t}\right\}^{2} d x$; that is, depends on $u_{x x t}$ and $u_{n x x t}$. However, it appears quite likely in many cases that $A_{n}^{*}$ will remain bounded as $n \rightarrow \infty$. In any case the inequality

$$
\int\left\{\left(u_{n}-u\right)_{x}\right\}^{2} d x \leq G_{n}^{*}(t)
$$

is a valid one, and in a given computation $G_{n}^{*}(t)$ can actually be computed. Setting $\Delta_{n}(x, t)=u_{n}-u$, we have from Schwarz's inequality

$$
\begin{aligned}
\left|\Delta_{n}(x, t)\right| & =\int_{0}^{x} \Delta_{n x}(s, t) d s \leq \sqrt{x \int^{x}\left\{\Delta_{n x}(s, t)\right\}^{2}} d s \\
& \leq \sqrt{x \mathcal{x}\left\{\Delta_{n x}(s, t)\right\}^{2} d s} \leq \sqrt{x G_{n}(t) .}
\end{aligned}
$$

In an analogous fashion we obtain by integrating from $\pi$ instead of from zero,

$$
\Delta_{n}(x, t) \leq \sqrt{(\pi-x) G_{n}^{*}(t)} .
$$

From (46) and (47) it follows that

$$
\Delta_{n}(x, t) \leq \sqrt{\frac{2}{\pi} x(\pi-x) G_{n}^{*}(t)} .
$$

Theorem 11.

$$
\left|u_{n}(x, t)-u(x, t)\right| \leq \sqrt{\frac{2}{\pi} x(\pi-x) G_{n}^{*}(t)},
$$

where

$$
\begin{aligned}
& G_{n}^{*}(t)=t F_{n}^{*}(t)\left\{A_{n}^{*}(t)+F_{n}^{*}(t)\right\}, \\
& F_{n}^{*}(t)=\max _{r \leq t} \sqrt{\int\left\{f_{n}-f\right\}^{2}} d x, \\
& A_{n}^{*}(t)-F_{n}^{*}(0)-t H_{n}^{*}(t), \\
& \left\{H_{n}^{*}(t)\right\}^{2}=G_{1}(t) t^{2}\left\{F_{n}^{*}(t)\right\}^{2}+2 G_{1}(t) t F_{n}^{*}(t)+F_{1 n}^{*}(t) \\
& \quad+\left\{F_{1 n}^{*}(t)\right\}^{2}, \\
& F_{1 n}^{*}(t)=\max _{r \leq t} \sqrt{\mathcal{S}\left\{f_{n t}(x, \tau)-f_{t}(x, \tau)\right\}^{2}} d x .
\end{aligned}
$$

For any stage $n$ of the solution, the function $G_{n}^{*}(t)$ can be explicitly calculated. If the $A_{n}^{*}$ do remain bounded, then $G_{n}^{*} \rightarrow 0$, and we will get a useful estimate of the error.

Los Angeles, April 24, 1952. 